\title{
KOSTKA-SHOJI POLYNOMIALS AND LUSZTIG'S CONVOLUTION DIAGRAM
}

\author{
MICHAEL FINKELBERG ${ }^{1, a}$ AND ANDREI IONOV ${ }^{2, b}$
}

To George Lusztig on his 70th birthday, with admiration

\footnotetext{
${ }^{1}$ National Research University Higher School of Economics, Russian Federation, Department of Mathematics, 6 Usacheva st, Moscow 119048; Institute for Information Transmission Problems of RAS; Skolkovo Institute of Science and Technology.

${ }^{a}$ E-mail: fnklberg@gmail.com

${ }^{2}$ National Research University Higher School of Economics, Russian Federation, Department of Mathematics, 6 Usacheva st, Moscow 119048.

${ }^{b}$ E-mail: 8916456@rambler.ru
}

\|\|

Abstract

We propose an $r$-variable version of Kostka-Shoji polynomials $K_{\lambda \mu}^{-}$for $r$-multipartitions $\boldsymbol{\lambda}, \boldsymbol{\mu}$. Our version has positive integral coefficients and encodes the graded multiplicities in the space of global sections of a line bundle over Lusztig's iterated convolution diagram for the cyclic quiver $\tilde{A}_{r-1}$.

\section{Introduction}

Let $G$ be a reductive complex algebraic group. According to G. Lusztig [9], the IC-stalks of $G[[z]]$-orbit closures in the affine Grassmannian $\operatorname{Gr}_{G}$ are encoded by the Kostka polynomials associated to the Langlands dual group $G^{\vee}$. According to R. Brylinski [4], the same Kostka polynomials encode the graded multiplicities in the global sections of line bundles on the cotangent bundle of the flag variety of $G^{\vee}$. According to [2], [5], the IC-stalks of $G L_{N}[[z]]$-orbit closures in the mirabolic affine Grassmannian of $G L_{N}$ are encoded by the Kostka-Shoji polynomials [16]. We note that the same KostkaShoji polynomials encode the graded multiplicities in the global sections of

Received May 8, 2016 and in revised form September 12, 2016.

AMS Subject Classification: 05E15 (14M15, 13A35).

Key words and phrases: Kostka-Shoji polynomials, cyclic quiver, convolution diagram, Frobenius splitting, affine flag variety, Bott-Samelson-Demazure-Hansen resolution. 
line bundles on a certain vector bundle over the square of the flag variety of $G L_{N}$. This vector bundle is nothing but Lusztig's iterated convolution diagram for the cyclic $\tilde{A}_{1}$ quiver [11]. The higher cohomology vanishing of the above line bundles follows from the Frobenius splitting of this convolution diagram which in turn follows from the fact that the convolution diagram is related to a Bott-Samelson-Demazure-Hansen (BSDH for short) variety of affine type $A[10]$.

The dilation action of $\mathbb{G}_{m}$ on Lusztig's convolution diagram extends to an action of $\mathbb{G}_{m} \times \mathbb{G}_{m}$ which gives rise to a 2 -variable version of Kostka-Shoji polynomials $K_{\boldsymbol{\lambda} \boldsymbol{\mu}}\left(t_{1}, t_{2}\right)$ such that $K_{\boldsymbol{\lambda} \boldsymbol{\mu}}(t, t)=K_{\boldsymbol{\lambda} \boldsymbol{\mu}}(t)$ (the classical KostkaShoji polynomial). Note that the realization of Kostka-Shoji polynomials via the IC-stalks on mirabolic affine Grassmannian cannot give rise to a 2-variable version since these stalks are pure Tate [2], [18], [5].

The (multi)graded multiplicities in the global sections of line bundles on Lusztig's iterated convolution diagram for the cyclic $\tilde{A}_{r-1}$ quiver are encoded conjecturally by an $r$-variable version of Kostka-Shoji polynomials $K_{\boldsymbol{\lambda} \boldsymbol{\mu}}^{-}(t)$ [16] for $r$-multipartitions $\boldsymbol{\lambda}, \boldsymbol{\mu}$. The higher cohomology vanishing is proved by the same argument as above. It would be interesting to find out if Lusztig's convolution diagrams for more general quivers are Frobenius split.

We are grateful to P. Achar, R. Bezrukavnikov, A. Braverman, B. Feigin, V. Ginzburg, S. Kato, A. Kuznetsov, L. Rybnikov, V. Serganova, T. Shoji, R. Travkin, M. Vikulina, L. Yanushevich for the helpful discussions. The work of M. F. was supported by the Russian Foundation for Sciences (project no. 14-50-00150) and carried out at the Institute for Information Transmission Problems of the Russian Academy of Sciences. The study of A. I. has been funded by the Russian Academic Excellence Project '5-100'. A. I. was supported in part by Dobrushin stipend and grant RFBR 15-01-09242.

\section{Kostka-Shoji Polynomials}

\subsection{Dominance order on multipartitions}

We denote by $\mathcal{P}_{N}^{r} \subset \mathbb{Z}^{r N}$ the set of generalized $r$-multipartitions $\boldsymbol{\lambda}=$ $\left(\lambda^{(1)}, \ldots, \lambda^{(r)}\right)$ such that for any $s=1, \ldots, r$ the corresponding $\lambda^{(s)}=\left(\lambda_{1}^{(s)} \geq\right.$ $\lambda_{2}^{(s)} \geq \cdots \geq \lambda_{N}^{(s)}$ ) is a weakly decreasing sequence of integers of length $N$. 
We order the entries of $\boldsymbol{\lambda}$ lexicographically as follows:

$$
\lambda_{1}^{(1)}, \lambda_{1}^{(2)}, \ldots, \lambda_{1}^{(r)}, \lambda_{2}^{(1)}, \lambda_{2}^{(2)}, \ldots, \lambda_{2}^{(r)}, \ldots, \lambda_{N}^{(r-1)}, \lambda_{N}^{(r)} .
$$

For any $n=1, \ldots, r N$ we denote by $\Sigma_{n}(\boldsymbol{\lambda})$ the sum of the first $n$ entries in the above order. We say that $\boldsymbol{\lambda} \geq \boldsymbol{\mu}$ in the dominance order if $\Sigma_{n}(\boldsymbol{\lambda}) \geq \Sigma_{n}(\boldsymbol{\mu})$ for any $n=1, \ldots, r N-1$, and $\Sigma_{r N}(\boldsymbol{\lambda})=\Sigma_{r N}(\boldsymbol{\mu})$. If $\boldsymbol{\lambda} \geq \boldsymbol{\mu}$, then $\alpha:=$ $\boldsymbol{\lambda}-\boldsymbol{\mu} \in \mathbb{N}^{r N-1}$ is the vector with coordinates $\left(\Sigma_{n}(\boldsymbol{\lambda})-\Sigma_{n}(\boldsymbol{\mu})\right)_{n=1, \ldots, r N-1}$.

\subsection{Partition function}

Let $\delta_{n}, 1 \leq n \leq r N-1$, be the base of $\mathbb{N}^{r N-1}$. For $1 \leq m<n \leq r N$ we set $\alpha_{m n}:=\sum_{l=m}^{n-1} \delta_{l}$. We define a finite subset $R_{r}^{+} \subset \mathbb{N}^{r N-1}$ of positive pseudoroots as follows: $R_{r}^{+}:=\left\{\alpha_{m n}\right\}_{n-m=1}(\bmod r)$.

Given $\alpha \in \mathbb{N}^{r N-1}$ we define a polynomial $L_{r}^{\alpha}(t)$ (Lusztig's partition function) as follows: $L_{r}^{\alpha}(t):=\sum p_{d} t^{d}$ where $p_{d}$ is the number of (unordered) partitions of $\alpha$ into a sum of $d$ positive pseudoroots. We extend $L_{r}(t)$ from $\mathbb{N}^{r N-1}$ to $\mathbb{Z}^{r N-1}$ by zero.

We also introduce a multivariable version of $L_{r}^{\alpha}\left(t_{1}, \ldots, t_{r}\right)$ where the variables are numbered by $\mathbb{Z} / r \mathbb{Z}=\{1, \ldots, r\}$. Namely, $L_{r}^{\alpha}\left(t_{1}, \ldots, t_{r}\right)=$ $\sum p_{\underline{d}} \prod_{s \in \mathbb{Z} / r \mathbb{Z}} t_{s}^{d_{s}}$ where $\underline{d}=\left(d_{1}, \ldots, d_{r}\right) \in \mathbb{N} \mathbb{Z} / r \mathbb{Z}$, and $p_{\underline{d}}$ is the number of unorderd partitions of $\alpha$ into a sum of positive pseudoroots having $d_{s}$ summands $\alpha_{m n}$ with $m=d_{s}(\bmod r)$ for any $s \in \mathbb{Z} / r \mathbb{Z}$. Clearly, the restriction of $L_{r}^{\alpha}\left(t_{1}, \ldots, t_{r}\right)$ to the diagonal $t_{1}=\ldots=t_{r}=t$ coincides with $L_{r}^{\alpha}(t)$. We extend $L_{r}\left(t_{1}, \ldots, t_{r}\right)$ from $\mathbb{N}^{r N-1}$ to $\mathbb{Z}^{r N-1}$ by zero.

\subsection{Lusztig-Kato formula}

We set $\rho=(N, N-1, \ldots, 2,1)$, and $\rho=(\rho, \ldots, \rho) \in \mathcal{P}_{N}^{r}$. Given $\boldsymbol{\lambda}, \boldsymbol{\mu} \in \mathcal{P}_{N}^{r}$ we define $K_{\boldsymbol{\lambda} \boldsymbol{\mu}}(t):=\sum_{\boldsymbol{\sigma} \in \mathfrak{S}_{N}^{r}}(-1)^{\boldsymbol{\sigma}} L_{r}^{\boldsymbol{\sigma}(\boldsymbol{\lambda}+\boldsymbol{\rho})-\boldsymbol{\rho}-\boldsymbol{\mu}}(t)$, the sum over the product of $r$ copies of the symmetric group $\mathfrak{S}_{N}$ acting on $\left(\mathbb{Z}^{N}\right)^{r}$ by permutations of entries of each composition.

We also introduce a multivariable version

$$
K_{\lambda \boldsymbol{\mu}}\left(t_{1}, \ldots, t_{r}\right):=\sum_{\boldsymbol{\sigma} \in \mathfrak{S}_{N}^{r}}(-1)^{\boldsymbol{\sigma}} L_{r}^{\boldsymbol{\sigma}(\boldsymbol{\lambda}+\boldsymbol{\rho})-\boldsymbol{\rho}-\boldsymbol{\mu}}\left(t_{1}, \ldots, t_{r}\right)
$$


Clearly, $K_{\boldsymbol{\lambda} \mu}(t, \ldots, t)=K_{\boldsymbol{\lambda} \boldsymbol{\mu}}(t)$.

Recall the Kostka-Shoji polynomials $K_{\lambda \mu}^{ \pm}(t)$ [16, 3.1]. In case $r=$ $1, K_{\lambda \mu}^{+}(t)=K_{\lambda \mu}^{-}(t)$ is the classical Kostka polynomial, and it was proved by I. G. Macdonald [12, page 243] that $K_{\lambda \mu}^{+}(t)=K_{\lambda \mu}^{-}(t)=K_{\lambda \mu}(t)$ for $\lambda \geq \mu$. ${ }^{1} \llbracket$ In case $r=2$, the identity $K_{\boldsymbol{\lambda} \boldsymbol{\mu}}^{+}(t)=K_{\boldsymbol{\lambda} \boldsymbol{\mu}}^{-}(t)=K_{\boldsymbol{\lambda} \boldsymbol{\mu}}(t)$ for $\boldsymbol{\lambda} \geq \boldsymbol{\mu}$ was proved by T. Shoji [16, Proof of Proposition 3.3]. The following generalization of these identities for arbitrary $r$ is supported by the calculations by L. Yanushevich for multipartitions of total size $\leq 7$, using P. Achar's code [1].

Conjecture 2.4. For multipartitions $\boldsymbol{\lambda} \geq \boldsymbol{\mu} \in \mathcal{P}_{N}^{r}$ we have $K_{\boldsymbol{\lambda} \boldsymbol{\mu}}^{-}(t)=$ $K_{\lambda \mu}(t)$.

\section{Lusztig's Convolution Diagram}

\subsection{A vector bundle over a flag variety}

We consider the following ordered base of an $r N$-dimensional vector space $\mathbb{C}^{r N}: v_{1}^{(1)}, \ldots, v_{1}^{(r)}, v_{2}^{(1)}, \ldots, v_{2}^{(r)}, \ldots, v_{N}^{(1)}, \ldots, v_{N}^{(r)}$. Sometimes, for $1 \leq$ $s \leq r, 1 \leq j \leq N$, we denote $v_{j}^{(s)}$ by $v_{r(j-1)+s}$. It gives rise to an embedding $G L_{N}^{r} \hookrightarrow G L_{r N}$ ( $s$-th copy of $G L_{N}$ acts in the summand spanned by $\left.v_{1}^{(s)}, \ldots, v_{N}^{(s)}\right)$, and also to an embedding of the Borel upper triangular subgroups $B_{N}^{r} \hookrightarrow B_{r N}$. In the adjoint representation of $G L_{r N}$ restricted to $B_{N}^{r}$ we consider a subrepresentation $\mathfrak{n}_{r}$ ( of $B_{N}^{r}$ ) spanned by the elementary matrices $E_{m n}, 1 \leq m<n \leq r N$ such that $n-m=1(\bmod r)$. It gives rise to a $G L_{N}^{r}$-equivariant vector bundle $\mathcal{T}_{r}^{*} \mathcal{B}_{N}^{r}=G L_{N}^{r} \times{ }^{B_{N}^{r}} \mathfrak{n}_{r}$ over the flag variety $\mathcal{B}_{N}^{r}$ of $G L_{N}^{r}$. Note that when $r=1$, the vector bundle $\mathcal{T}_{1}^{*} \mathcal{B}_{N}$ over the flag variety $\mathcal{B}_{N}$ is nothing but the cotangent bundle.

Let $x_{1}, \ldots, x_{r N}$ stand for the characters of the diagonal Cartan torus $T_{r N}$ of $G L_{r N}$ corresponding to the diagonal matrix entries. Sometimes, for $1 \leq s \leq r, 1 \leq j \leq N$, we denote $x_{r(j-1)+s}$ by $x_{j}^{(s)}$. For $1 \leq m<n \leq$ $r N$ we set $x^{\alpha_{m n}}=x_{m}^{-1} x_{n}$. This is the weight of the elementary matrix $E_{n m}$. This rule extends to a homomorphism $\mathbb{N}^{r N-1} \rightarrow X^{*}\left(T_{r N}\right), \alpha \rightarrow$ $x^{\alpha}$. The symmetric algebra $\operatorname{Sym}^{\bullet} \mathfrak{n}_{r}^{\vee}$ is graded, and its character is a formal series in $x_{1}, \ldots, x_{r N}, t$. In fact, $\operatorname{Sym} \bullet \mathfrak{n}_{r}^{\vee}$ has a finer grading by $\mathbb{N} \mathbb{Z} / r \mathbb{Z}$ arising

\footnotetext{
${ }^{1}$ A similar identity for arbitrary finite root systems was conjectured by G. Lusztig [9, (9.4)] and proved by S.-I. Kato [7, Theorem 1.3].
} 
from a $\mathbb{Z} / r \mathbb{Z}$-grading of $\mathfrak{n}_{r}^{\vee}: \operatorname{deg} E_{n m}:=m(\bmod r)$. Hence the character of $\mathrm{Sym}^{\bullet} \mathfrak{n}_{r}^{\vee}$ is a formal series $\chi$ in $x_{1}, \ldots, x_{r N}, t_{1}, \ldots, t_{r}$.

Lemma 3.2. $\chi=\sum_{\alpha \in \mathbb{N}^{r N-1}} L^{\alpha}\left(t_{1}, \ldots, t_{r}\right) x^{\alpha}$.

Proof. Clear.

Given a multipartition $\boldsymbol{\mu} \in \mathcal{P}_{N}^{r}$, we consider the corresponding $G L_{N^{-}}^{r}$ equivariant line bundle $\mathcal{O}(\boldsymbol{\mu})$ on $\mathcal{B}_{N}^{r}$ : the action of $B_{N}^{r}$ on its fiber at the point $B_{N}^{r} \in \mathcal{B}_{N}^{r}$ is via the character $\prod\left(x_{j}^{(s)}\right)^{-\mu_{j}^{(s)}}$. Its global sections $\Gamma\left(\mathcal{B}_{N}^{r}, \mathcal{O}(\boldsymbol{\mu})\right)$ is an irreducible $G L_{N}^{r}$-module $V^{\boldsymbol{\mu}}$ with lowest weight $-\boldsymbol{\mu}$. The character of $V^{\boldsymbol{\mu}}$ will be denoted $\chi^{\boldsymbol{\mu}} \in \mathbb{Z}\left[x_{1}^{ \pm 1}, \ldots, x_{r N}^{ \pm 1}\right]$. The pullback of $\mathcal{O}(\boldsymbol{\mu})$ to $\mathcal{T}_{r}^{*} \mathcal{B}_{N}^{r}$ will be also denoted $\mathcal{O}(\boldsymbol{\mu})$ when no confusion is likely. We consider the equivariant Euler characteristic $\chi\left(\mathcal{T}_{r}^{*} \mathcal{B}_{N}^{r}, \mathcal{O}(\boldsymbol{\mu})\right)=\chi\left(\mathcal{B}_{N}^{r}, \operatorname{Sym}^{\bullet} \mathcal{T}_{r} \mathcal{B}_{N}^{r} \otimes \mathcal{O}(\boldsymbol{\mu})\right)$ where $\mathcal{T}_{r} \mathcal{B}_{N}^{r}=G L_{N}^{r} \times{ }^{B_{N}^{r}} \mathfrak{n}_{r}^{\vee}$ stands for the vector bundle over $\mathcal{B}_{N}^{r}$ dual to $\mathcal{T}_{r}^{*} \mathcal{B}_{N}^{r}$. The $\mathbb{N} \mathbb{Z} / r \mathbb{Z}$-grading of $\operatorname{Sym}^{\bullet} \mathfrak{n}_{r}^{\vee}$ gives rise to a $\mathbb{N} \mathbb{Z} / r \mathbb{Z}$-grading of $\operatorname{Sym}^{\bullet} \mathcal{T}_{r} \mathcal{B}_{N}^{r} \otimes \mathcal{O}(\boldsymbol{\mu})$, and hence $\chi\left(\mathcal{B}_{N}^{r}, \operatorname{Sym}^{\bullet} \mathcal{T}_{r} \mathcal{B}_{N}^{r} \otimes \mathcal{O}(\boldsymbol{\mu})\right)$ is a formal series in $x_{1}, \ldots, x_{r N}, t_{1}, \ldots, t_{r}$.

Corollary 3.3. $\chi\left(\mathcal{B}_{N}^{r}, \operatorname{Sym}^{\bullet} \mathcal{T}_{r} \mathcal{B}_{N}^{r} \otimes \mathcal{O}(\boldsymbol{\mu})\right)=\sum_{\boldsymbol{\lambda} \geq \boldsymbol{\mu}} K_{\boldsymbol{\lambda} \boldsymbol{\mu}}\left(t_{1}, \ldots, t_{r}\right) \chi^{\boldsymbol{\lambda}}$.

Proof. Same as the proof of [4, Lemma 6.1].

\subsection{Convolution diagram}

Recall the notations of [11, Section 1]. We consider the type $\tilde{A}_{r-1}$ cyclic quiver $Q$ with the set $\mathbb{Z} / r \mathbb{Z}$ of vertices, and with arrows $s \rightarrow s-$ $1, s \in \mathbb{Z} / r \mathbb{Z}$. Let $\mathbf{V}$ be a $\mathbb{Z} / r \mathbb{Z}$-graded vector space such that $\operatorname{dim} \mathbf{V}_{s}=$ $N$ for any $s \in \mathbb{Z} / r \mathbb{Z}$. Let $\mathbf{i}$ be a length $r N$ periodic sequence $(r, r-$ $1, \ldots, 2,1, r, r-1, \ldots, 2,1, \ldots, r, \ldots, 1)$ of vertices, and let a be a length $r N$ sequence $(1,1, \ldots, 1)$ of positive integers. Then the variety $\mathcal{F}_{\mathbf{i}, \mathbf{a}}$ of all flags of type $(\mathbf{i}, \mathbf{a})$ in $\mathbf{V}$ is nothing but $\mathcal{B}_{N}^{r}$. Moreover, the iterated convolution diagram $\widetilde{\mathcal{F}}_{\mathbf{i}, \mathbf{a}}$ of $[11,1.5]$ is nothing but $\mathcal{T}_{r}^{*} \mathcal{B}_{N}^{r}$. In effect, we identify $\mathbf{V}_{s}$ with a vector subspace of $\mathbb{C}^{r N}$ spanned by $v_{1}^{(s)}, \ldots, v_{N}^{(s)}$ (notations of 3.1). Then the fiber of the natural $G L(\mathbf{V})=G L_{N}^{r}$-equivariant projection $\widetilde{\mathcal{F}}_{\mathbf{i}, \mathbf{a}} \rightarrow \mathcal{F}_{\mathbf{i}, \mathbf{a}}$ over the flag $\mathbf{V}=\mathbb{C} v_{1}^{(1)} \oplus \ldots \oplus \mathbb{C} v_{N}^{(r)} \supset \mathbb{C} v_{1}^{(1)} \oplus \ldots \oplus v_{N}^{(r-1)} \supset \ldots \supset \mathbb{C} v_{1}^{(1)} \oplus$ $\ldots \oplus \mathbb{C} v_{1}^{(r)} \oplus \mathbb{C} v_{2}^{(1)} \supset \mathbb{C} v_{1}^{(1)} \oplus \ldots \oplus \mathbb{C} v_{1}^{(r)} \supset \ldots \supset \mathbb{C} v_{1}^{(1)} \oplus \mathbb{C} v_{1}^{(2)} \supset \mathbb{C} v_{1}^{(1)} \supset 0$ is nothing but $\mathfrak{n}_{r}$. 


\section{Frobenius Splitting of $\mathcal{T}_{r}^{*} \mathcal{B}_{N}^{r}$}

In this section we replace $\mathbb{C}$ by an algebraic closure $k$ of the finite field $\mathbb{F}_{p}$ of characteristic $p$. The present section is devoted to the proof of the following

Theorem 4.1. $\mathcal{T}_{r}^{*} \mathcal{B}_{N}^{r}$ is Frobenius split.

Our proof is a variation of the one in [14].

\subsection{The canonical bundle of $\mathcal{T}_{r}^{*} \mathcal{B}_{N}^{r}$}

We have a subgroup $S L_{N}^{r} \subset G L_{N}^{r}$ (product of $r$ copies of $S L_{N}$ ).

Lemma 4.3. The canonical line bundle $\omega$ of $\mathcal{T}_{r}^{*} \mathcal{B}_{N}^{r}$ is $S L_{N}^{r}$-equivariantly trivial.

Proof. The product $w_{1}$ of $T_{r N}$-weights in $\mathfrak{n}_{r}$ is

$$
\begin{aligned}
& \prod_{s=2}^{r} \prod_{1 \leq k \leq l \leq N} x_{r(k-1)+s-1} x_{r(l-1)+s}^{-1} \cdot \prod_{1 \leq k<l \leq N} x_{r k} x_{r(l-1)+1}^{-1} \\
& \quad=\prod_{s=1}^{r} \prod_{k=1}^{N} x_{r(k-1)+s}^{N+1-2 k} \cdot \prod_{k=1}^{N} x_{r k} x_{r(k-1)+1}^{-1} \cdot
\end{aligned}
$$

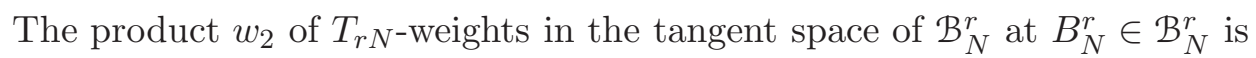

$$
\prod_{s=1}^{r} \prod_{N \geq k>l \geq 1} x_{r(k-1)+s} x_{r(l-1)+s}^{-1}=\prod_{s=1}^{r} \prod_{k=1}^{N} x_{r(k-1)+s}^{2 k-N-1} .
$$

The $T_{r N}$-weight in the fiber of the canonical bundle at the point $B_{N}^{r} \in$ $\mathcal{B}_{N}^{r} \subset \mathcal{T}_{r}^{*} \mathcal{B}_{N}^{r}$ is $w=w_{1}^{-1} w_{2}^{-1}=\prod_{k=1}^{N} x_{r k}^{-1} x_{r(k-1)+1}$. When we restrict $w$ to the maximal torus of $S L_{N}^{r}$ we get the trivial weight, hence the canonical line bundle $\omega$ is $S L_{N}^{r}$-equivariantly trivial.

\subsection{A splitting section}

According to [3, Theorem 1.3.8], in order to prove the Frobenius splitting of $\mathcal{T}_{r}^{*} \mathcal{B}_{N}^{r}$ it suffices to construct a section $\phi \in \Gamma\left(\mathcal{T}_{r}^{*} \mathcal{B}_{N}^{r}, \omega^{1-p}\right)$ in whose expansion with respect to some local coordinates $z_{1}, \ldots, z_{d}$ the monomial 
$z_{1}^{p-1} \cdots z_{d}^{p-1}$ occurs with coefficient 1 . Since $\omega$ is $S L_{N}^{r}$-equivariantly trivial, it has an $S L_{N}^{r}$-invariant nowhere vanishing section $\varpi$, and we will look for the desired section $\phi$ in the form $\phi=f \varpi^{1-p}$ for some $f \in \mathrm{k}\left[\mathcal{T}_{r}^{*} \mathcal{B}_{N}^{r}\right]$.

To this end recall the decomposition $\mathrm{k}^{r N}=\bigoplus_{1 \leq s \leq r} \mathbf{V}_{s}$ of Section 3.4. Accordingly, we will write down the matrices $A \in \mathfrak{g l}_{r N}$ in the block form

$$
\left(\begin{array}{cccc}
A_{11} & A_{12} & \ldots & A_{1 r} \\
A_{21} & A_{22} & \ldots & A_{1 n} \\
\vdots & \vdots & \ddots & \vdots \\
A_{r 1} & A_{r 2} & \ldots & A_{r r}
\end{array}\right)
$$

(for $1 \leq s, u \leq r$ the corresponding block $A_{s u}$ is an $N \times N$-matrix). The subgroup $B_{N}^{r} \subset G L_{N}^{r} \subset G L_{r N}$ is formed by all the matrices with uppertriangular diagonal blocks and vanishing non-diagonal blocks. The subspace $\mathfrak{n}_{r} \subset \mathfrak{g l}_{r N}$ is formed by all the matrices with strictly upper triangular block $A_{r 1}$, nonstrictly upper triangular blocks $A_{s, s+1}, 1 \leq s \leq r-1$, and all the other blocks vanishing. Hence $\mathcal{T}_{r}^{*} \mathcal{B}_{N}^{r}=G L_{N}^{r} \times B_{N}^{r} \mathfrak{n}_{r}$ is the quotient of $G L_{N}^{r} \times$ $\mathfrak{n}_{r}=\left\{\left(g_{1}, \ldots, g_{r} ; A_{12}, A_{23}, \ldots, A_{r-1, r}, A_{r 1}\right)\right\}$ modulo the action of $B_{N}^{r}=$ $\left\{\left(b_{1}, \ldots, b_{r}\right)\right\}$ given by $\left(b_{1}, \ldots, b_{r}\right) \cdot\left(g_{1}, \ldots, g_{r} ; A_{12}, A_{23}, \ldots, A_{r-1, r}, A_{r 1}\right)=$ $\left(g_{1} b_{1}^{-1}, \ldots, g_{r} b_{r}^{-1} ; b_{1} A_{12} b_{2}^{-1} \ldots, b_{r-1} A_{r-1, r} b_{r}^{-1}, b_{r} A_{r 1} b_{1}^{-1}\right)$. We define

$$
\begin{aligned}
& f\left(g_{1}, \ldots, g_{r} ; A_{12}, A_{23}, \ldots, A_{r-1, r}, A_{r 1}\right) \\
& \quad:=\prod_{s=1}^{r-1} \prod_{j=1}^{N} \Delta_{j}\left(g_{s} A_{s, s+1} g_{s+1}^{-1}\right) \cdot \prod_{j=1}^{N-1} \Delta_{j}\left(g_{r} A_{r 1} g_{1}^{-1}\right)
\end{aligned}
$$

where $\Delta_{j}$ stands for the principal $j \times j$-minor in the upper left corner.

Proposition 4.5. The section $\phi=f^{p-1} \varpi^{1-p} \in \Gamma\left(\mathcal{T}_{r}^{*} \mathcal{B}_{N}^{r}, \omega^{1-p}\right)$ splits $\mathcal{T}_{r}^{*} \mathcal{B}_{N}^{r}$.

The proof is given in Section 4.8 after a preparation in Section 4.6.

\subsection{Residues}

We recall the following construction [14, 3.5]. Given a smooth divisor $Z$ in a smooth variety $Y$ and a global section of the anticanonical class $\eta \in$ $\Gamma\left(Y, \omega_{Y}^{-1}\right)$ we construct the residue res $\eta \in \Gamma\left(Z, \omega_{Z}^{-1}\right)$ as follows. We start with an open subvariety $U \subset Z$ such that the normal bundle $\left.\mathcal{N}_{Z / Y}\right|_{U}$ restricted to 
$U$ is trivial. We choose a nowhere vanishing section $\sigma \in \Gamma\left(U,\left.\mathcal{N}_{Z / Y}\right|_{U}\right)$. Then $\left.\operatorname{res} \eta\right|_{U}$ is defined by the requirement $\langle\operatorname{res} \eta, \zeta\rangle=\left\langle\eta, \zeta \frac{d \sigma}{\sigma}\right\rangle$ for any $\zeta \in \Gamma\left(U, \omega_{U}\right)$ where $\langle$,$\rangle is the pairing between the anticanonical and canonical bundles.$ One can check that res $\left.\eta\right|_{U}$ is independent of the choice of $\sigma$, and for $U^{\prime} \subset U$ we have $\left.\left(\left.\operatorname{res} \eta\right|_{U}\right)\right|_{U^{\prime}}=\left.\operatorname{res} \eta\right|_{U^{\prime}}$, so the local sections res $\left.\eta\right|_{U}$ glue to the desired res $\eta$. If we have a chain of smooth divisors $Y \supset Z_{1} \supset \cdots \supset Z_{n}$ we can iterate the above construction to obtain res: $\Gamma\left(Y, \omega_{Y}^{-1}\right) \rightarrow \Gamma\left(Z_{n}, \omega_{Z_{n}}^{-1}\right)$.

Lemma 4.7. There is a chain of smooth divisors $\mathcal{T}_{r}^{*} \mathcal{B}_{N}^{r}=Y \supset Z_{1} \supset \cdots \supset$ $Z_{n}=\mathcal{B}_{N}^{r}$ such that $\left(\operatorname{res} f \varpi^{-1}\right)^{p-1} \in \Gamma\left(\mathcal{B}_{N}^{r}, \omega_{\mathcal{B}_{N}^{r}}^{-1}\right)$ gives rise to a Frobenius splitting of $\mathcal{B}_{N}^{r}$ compatible with the splitting $\varphi: \operatorname{Fr}_{*} \mathcal{O}_{\mathcal{B}_{N}^{r}} \rightarrow \mathcal{O}_{\mathcal{B}_{N}^{r}}$ arising from $f^{p-1} \varpi^{1-p}$.

Proof. It suffices to argue generically on $\mathcal{B}_{N}^{r}$. Let $X_{N} \subset \mathcal{B}_{N}$ be an open Bruhat cell: the open orbit of the strictly lower triangular subgroup $U_{N}^{-} \subset$ $G L_{N}$. We consider an open cell $X:=X_{N}^{r} \times \mathfrak{n}_{r} \subset \mathcal{T}_{r}^{*} \mathcal{B}_{N}^{r}$; we have $X \cap \mathcal{B}_{N}^{r}=$ $X_{N}^{r}$. We will calculate residues on $X$, so in the definition (4.1) of the function $f$ we will assume that $g_{s}$ are strictly lower triangular for any $1 \leq s \leq r$. But for $g \in U_{N}^{-}$and $A \in \mathfrak{g l}_{N}$ we have $\Delta_{j}(g A)=\Delta_{j}(A)$ for any $1 \leq j \leq N$. Hence $\Delta_{j}\left(g_{s} A_{s, s+1} g_{s+1}^{-1}\right)=\Delta_{j}\left(A_{s, s+1} g_{s+1}^{-1}\right)=\Delta_{j}\left(g_{s+1} A_{s, s+1} g_{s+1}^{-1}\right)$. Therefore, we can identify $X \subset \mathcal{T}_{r}^{*} \mathcal{B}_{N}^{r}$ with $\hat{X}:=\left(U_{N}^{-} \times \mathfrak{b}_{N}\right)^{r-1} \times\left(U_{N}^{-} \times \mathfrak{u}_{N}\right) \subset\left(G L_{N} \times B_{N}\right.$ $\left.\mathfrak{b}_{N}\right)^{r-1} \times\left(G L_{N} \times{ }^{B_{N}} \mathfrak{u}_{N}\right)\left(\right.$ where $\mathfrak{b}_{N} \supset \mathfrak{u}_{N}$ is the Lie algebra of $B_{N}$ and its nilpotent radical) so that $\left.f \varpi^{-1}\right|_{X}=\left.\hat{f} \hat{\varpi}^{-1}\right|_{\hat{X}}$. Here $\hat{\varpi}$ is an $S L_{N}^{r}$-invariant nowhere vanishing volume form on $\hat{X}$, and

$$
\hat{f}\left(g_{1}, A_{1}, \ldots, g_{r}, A_{r}\right):=\prod_{s=1}^{r} \prod_{j=1}^{N} \Delta_{j}\left(g_{s} A_{s} g_{s}^{-1}\right) .
$$

According to [14, Theorem 3.8], the chains of divisors required in the lemma exist for each factor $\hat{X}_{s}$ of $\hat{X}$ (that is, $G L_{N} \times{ }^{B_{N}} \mathfrak{b}_{N} \supset \mathcal{B}_{N}$ or $G L_{N} \times{ }^{B_{N}} \mathfrak{u}_{N} \supset$ $\left.\mathcal{B}_{N}\right)$ equipped with the function $\hat{f}_{s}:=\prod_{j=1}^{N} \Delta_{j}\left(g_{s} A_{s} g_{s}^{-1}\right)$ and section $\hat{\varpi}_{s}$, and the required compatibilities hold. Hence the desired compatibility holds for their external product.

\subsection{Proof of Proposition 4.5}

The section $f^{p-1} \varpi^{1-p} \in \Gamma\left(\mathcal{T}_{r}^{*} \mathcal{B}_{N}^{r}, \omega^{1-p}\right)$ gives rise to a morphism $\varphi$ : $\operatorname{Fr}_{*} \mathcal{O}_{\mathcal{T}_{r}^{*} \mathcal{B}_{N}^{r}} \rightarrow \mathcal{O}_{\mathcal{T}_{r}^{*} \mathcal{B}_{N}^{r}}$, and we have to check that the composition $\mathcal{O}_{\mathcal{T}_{r}^{*} \mathcal{B}_{N}^{r}} \rightarrow$ 
$\operatorname{Fr}_{*} \mathcal{O}_{\mathcal{T}_{r}^{*} \mathcal{B}_{N}^{r}} \rightarrow \mathcal{O}_{\mathcal{T}_{r}^{*} \mathcal{B}_{N}^{r}}$ is the identity morphism. It suffices to check $\varphi(1)=1$. We know from Lemma 4.7 that $\left.\varphi(1)\right|_{\mathcal{B}_{N}^{r}}=1$.

We consider a one-parametric central subgroup $\mathbb{G}_{m} \hookrightarrow G L_{N}^{r}$ whose $s$-th component is $t \mapsto t^{s}$ for $1 \leq s \leq r$. Then $f \varpi^{-1}$ is a $\mathbb{G}_{m}$-eigensection with a nontrivial character. Hence the zero divisor of $\varphi(1)$ is $\mathbb{G}_{m}$-invariant. Since $\left(\mathcal{T}_{r}^{*} \mathcal{B}_{N}^{r}\right)^{\mathbb{G}_{m}}=\mathcal{B}_{N}^{r}$ and $\left.\varphi(1)\right|_{\mathcal{B}_{N}^{r}}=1$, the function $\varphi(1)$ has an empty zero divisor. Since the fibers of the projection $\mathcal{T}_{r}^{*} \mathcal{B}_{N}^{r} \rightarrow \mathcal{B}_{N}^{r}$ are vector spaces, and a nowhere vanishing function on a vector space is constant, we conclude $\varphi(1)=1$.

\section{Frobenius Splitting of Lusztig's Convolution Diagrams}

The aim of this Section is a proof of the following generalization of Theorem 4.1 .

Theorem 5.1. Let $\mathbf{i}$ be an arbitrary length $\ell$ sequence of vertices of the cyclic quiver $Q$. Let $\mathbf{a}$ be an arbitrary length $\ell$ sequence of positive integers. Then the iterated convolution diagram $\widetilde{\mathcal{F}}_{\mathbf{i}, \mathbf{a}}$ is Frobenius split.

Our proof follows [10, Section 11] covering $\widetilde{\mathcal{F}}_{\mathbf{i}, \mathbf{a}}$ by an open subvariety of an affine type $A$ BSDH resolution, and then applying Frobenius splitting for BSDH resolutions [13, Lemme 52].

\subsection{Recollections of [10], [11]}

We fix $\mathbf{d}=\left(d_{1}, \ldots, d_{r}\right) \in \mathbb{N}^{\mathbb{Z} / r \mathbb{Z}}$, and consider a $\mathbb{Z} / r \mathbb{Z}$-graded vector space $\mathbf{V}=\bigoplus_{s \in \mathbb{Z} / r \mathbb{Z}} \mathbf{V}_{s}$ such that $\operatorname{dim} \mathbf{V}_{s}=d_{s}$. Given a length $\ell$ sequence $\mathbf{i}=\left(s_{1}, \ldots, s_{\ell}\right) \in(\mathbb{Z} / r \mathbb{Z})^{\ell}$ and a length $\ell$ sequence $\mathbf{a}=\left(a_{1}, \ldots, a_{\ell}\right) \in \mathbb{N}^{\ell}$ such that $\sum_{n: s_{n}=s} a_{n}=d_{s}$ for any $s \in \mathbb{Z} / r \mathbb{Z}$, we consider the iterated convolution diagram $\widetilde{\mathcal{F}}_{\mathbf{i}, \mathbf{a}}=\left\{\left(\mathbf{V}^{\bullet}, f\right)\right\}$. Here $\mathbf{V}^{\bullet}$ is a $\mathbb{Z} / r \mathbb{Z}$-graded flag in $\mathbf{V}: \mathbf{V}=\mathbf{V}^{0} \supset \mathbf{V}^{1} \supset \cdots \supset \mathbf{V}^{\ell}=0$ such that $\mathbf{V}^{n-1} / \mathbf{V}^{n}$ is an $a_{n}$-dimensional vector space supported at the vertex $s_{n}$ for any $n=1, \ldots, \ell$, and $f=$ $\left(f_{s}: \mathbf{V}_{s} \rightarrow \mathbf{V}_{s-1}\right)_{s \in \mathbb{Z} / r \mathbb{Z}}$ is a $Q$-module structure on $\mathbf{V}$ such that $f \mathbf{V}^{n-1} \subset$ $\mathbf{V}^{n}$ for any $1 \leq n \leq \ell$. The convolution diagram $\widetilde{\mathcal{F}}_{\mathbf{i}, \mathbf{a}}$ is smooth, being a vector bundle over a flag variety of $G L(\mathbf{V})=\prod_{s \in \mathbb{Z} / r \mathbb{Z}} G L\left(\mathbf{V}_{s}\right)$. We have a projection $\pi: \widetilde{\mathcal{F}}_{\mathbf{i}, \mathbf{a}} \rightarrow \mathbf{E}_{\mathbf{V}},\left(\mathbf{V}^{\bullet}, f\right) \mapsto f$ to the vector space $\mathbf{E}_{\mathbf{V}}$ of $Q$-modules with underlying space $\mathbf{V}$. The morphism $\pi$ is proper, and its image is the 
closure $\overline{\mathbb{O}}_{\mathbf{i}, \mathbf{a}}$ of a nilpotent $G L(\mathbf{V})$-orbit in $\mathbf{E}_{\mathbf{V}}$. The union of all nilpotent $G L(\mathbf{V})$-orbits in $\mathbf{E}_{\mathbf{V}}$ is a closed subvariety $\mathbf{E}_{\mathbf{V}}^{\text {nil }} \subset \mathbf{E}_{\mathbf{V}}$ (possibly reducible).

Let $d:=d_{1}+\cdots+d_{r}$. Let $F$ be a $d$-dimensional vector space over the Laurent series field $\mathrm{k}((\epsilon))$. We fix a flag of lattices $\cdots \supset L_{-1} \supset L_{0} \supset L_{1} \supset \cdots$ in $F$ such that $L_{s+r}=\epsilon L_{s}, L_{s} \supset L_{s+1}$ and $\operatorname{dim}\left(L_{s} / L_{s+1}\right)=d_{s}(\bmod r)$ for any $s \in \mathbb{Z}$. We consider a type $\tilde{A}_{d-1}$ affine Schubert variety $Z$ formed by all the flags of lattices $\cdots \supset M_{-1} \supset M_{0} \supset M_{1} \supset \cdots$ in $F$ such that $M_{s+r}=$ $\epsilon M_{s}, M_{s} \supset M_{s+1}$ and $M_{s} \subset L_{s}, \operatorname{dim}\left(L_{s} / M_{s}\right)=d_{s}(\bmod r)$ for any $s \in \mathbb{Z}$. In [10, 11.4], G. Lusztig constructs an open dense embedding $\varphi: \mathbf{E}_{\mathbf{V}}^{\text {nil }} \hookrightarrow Z$ (the image $\stackrel{\circ}{Z}=\varphi\left(\mathbf{E}_{\mathbf{V}}^{\text {nil }}\right) \subset Z$ is specified by certain transversality conditions) such that for any nilpotent $G L(\mathbf{V})$-orbit closure $\overline{\mathbb{O}}_{\mathbf{i}, \mathbf{a}} \subset \mathbf{E}_{\mathbf{V}}^{\text {nil }}$ its image $\varphi\left(\overline{\mathbb{O}}_{\mathbf{i}, \mathbf{a}}\right)$ is the intersection of $\stackrel{\circ}{Z}$ with a Schubert subvariety $Z_{\mathbf{i}, \mathbf{a}} \subset Z$.

The construction of [10, 11.4] yields an isomorphism $\widetilde{\mathcal{F}}_{\mathbf{i}, \mathbf{a}} \simeq \stackrel{\circ}{Z}^{\times}{ }_{Z} \widetilde{Z}_{\mathbf{i}, \mathbf{a}}$ for a BSDH type resolution $\widetilde{Z}_{\mathbf{i}, \mathbf{a}} \rightarrow Z_{\mathbf{i}, \mathbf{a}}$ formed by all the collections $\left(M_{s}^{n}\right)_{s \in \mathbb{Z}}^{\substack{0 \leq n \leq \ell \\ \text { a }}}$ of double flags of lattices such that (a) $M_{s}^{0}=L_{s}$; (b) $M_{s}^{n} \supset M_{s+1}^{n}$ and $M_{s+r}^{n}=\epsilon M_{s}^{n}$; (c) $M_{s}^{n-1}=M_{s}^{n}$ unless $s=s_{n}(\bmod r) ;(\mathrm{d})$ if $s=s_{n}(\bmod r)$, then $M_{s}^{n-1} \supset M_{s}^{n}$, and $\operatorname{dim} M_{s}^{n-1} / M_{s}^{n}=a_{n}$.

\subsection{BSDH resolution}

According to [3, Lemma 1.4.5], in order to construct a Frobenius splitting of $\widetilde{Z}_{\mathbf{i}, \mathbf{a}}$ (and hence of its open subvariety $\widetilde{\mathcal{F}}_{\mathbf{i}, \mathbf{a}}$ ) it suffices to construct a proper dominant morphism $\varpi: \widehat{Z}_{\mathbf{i}, \mathbf{a}} \rightarrow \widetilde{Z}_{\mathbf{i}, \mathbf{a}}$ with connected fibers from a Frobenius split variety $\widehat{Z}_{\mathbf{i}, \mathbf{a}}$. We will construct $\varpi: \widehat{Z}_{\mathbf{i}, \mathbf{a}} \rightarrow \widetilde{Z}_{\mathbf{i}, \mathbf{a}}$ in two steps.

First we define $\widehat{Z}_{\mathbf{i}, \mathbf{a}}^{\prime}:=\widetilde{Z}_{\mathbf{i}, \mathbf{a}} \times_{Z} \mathcal{F} \ell_{Z}$ where $\mathcal{F} \ell_{Z}$ is formed by all the complete flags of lattices $\cdots \supset K_{-1} \supset K_{0} \supset K_{1} \supset \cdots$ (so that $K_{u+d}=$ $\epsilon K_{u}, K_{u} \supset K_{u+1}$ and $\operatorname{dim} K_{u} / K_{u+1}=1$ for any $u \in \mathbb{Z}$ ) such that for $s \geq 0, L_{s} \supset K_{d_{0}+d_{1}+\cdots+d_{s}}$ and $\operatorname{dim} L_{s} / K_{d_{0}+d_{1}+\cdots+d_{s}}=d_{s}$, while for $s<$ $0, L_{s} \supset K_{d_{0}-d_{-1}-\cdots-d_{s}}$ and $\operatorname{dim} L_{s} / K_{d_{0}-d_{-1}-\cdots-d_{s}}=d_{s}$. The evident projection $\mathcal{F} \ell_{Z} \rightarrow Z$ sends $K \bullet$ to $M_{\bullet}$ where for $s \geq 0, M_{s}=K_{d_{0}+d_{1}+\cdots+d_{s}}$, while for $s<0, M_{s}=K_{d_{0}-d_{-1}-\cdots-d_{s}}$. This projection is a fibration with a fiber isomorphic to a (finite) flag variety of type $A$.

Let us choose a base point $K_{\bullet}^{0} \in \mathcal{F} \ell_{Z}$ such that $K_{0}^{0}=L_{0}$, and for $s>$ $0, K_{d_{1}+\cdots+d_{s}}^{0}=L_{s}$, and for $s<0, K_{-d_{-1}-\cdots-d_{s}}^{0}=L_{s}$. Then the connected component $\mathcal{F} \ell$ of the ind-variety of complete flags of lattices containing $K_{\bullet}^{0}$ is identified with the affine flag variety of $S L_{d}$. The simple reflections of 
its affine Weyl group are numbered by $\mathbb{Z} / d \mathbb{Z}$, and any finite sequence $\underline{u}=$ $(u(1), u(2), \ldots, u(k)), u(j) \in \mathbb{Z} / d \mathbb{Z}$, gives rise to a BSDH variety $D_{\underline{u}} \rightarrow \mathcal{F} \ell$ projecting to $\mathcal{F} \ell$ with connected fibers.

We consider a concatenated sequence $\underline{u}=\left(\underline{u}_{\ell}, \ldots, \underline{u}_{1}\right)$ where for $1 \leq$ $n \leq \ell \underline{u}_{n}$ is a sequence of integers in the interval

$$
\left[d_{1}+\cdots+d_{s_{n}}+1+\sum_{m<n: s_{m}=s_{n}} a_{m}, d_{1}+\cdots+d_{s_{n}+1}-1+\sum_{m<n: s_{m}=s_{n}+1} a_{m}\right]
$$

giving a reduced expression of the longest element of the (finite) parabolic Weyl subgroup generated by the simple reflections numbered by (the residues modulo $d$ of) the integers in the above interval. Then there is a dominant projection $D_{\underline{u}} \rightarrow \widehat{Z}_{\mathbf{i}, \mathbf{a}}^{\prime}$ with connected fibers. Finally, $D_{\underline{u}}$ is Frobenius split according to [13, Lemme 52]. Theorem 5.1 is proved.

\section{Cohomology Vanishing}

We say that a generalized multipartition $\boldsymbol{\mu}=\left(\mu^{(1)}, \ldots, \mu^{(r)}\right) \in \mathcal{P}_{N}^{r}$ is regular if for any $s=1, \ldots, r$ we have $\mu_{1}^{(s)}>\mu_{2}^{(s)}>\cdots>\mu_{N}^{(s)}$. In this case the line bundle $\mathcal{O}(\boldsymbol{\mu})$ on $\mathcal{T}_{r}^{*} \mathcal{B}_{N}^{r}$ is very ample, and we deduce from Theorem 4.1 and [3, Lemma 1.2.7(i)] the following

Corollary 6.1. For a regular multipartition $\boldsymbol{\mu} \in \mathcal{P}_{N}^{r}$, we have the higher cohomology vanishing $H^{>0}\left(\mathcal{T}_{r}^{*} \mathcal{B}_{N}^{r}, \mathcal{O}(\boldsymbol{\mu})\right)=0$.

Similarly to [3, Theorem 5.2.12] we put forth the following

Conjecture 6.2. For a multipartition $\boldsymbol{\mu} \in \mathcal{P}_{N}^{r}$, we have the higher cohomology vanishing $H^{>0}\left(\mathcal{T}_{r}^{*} \mathcal{B}_{N}^{r}, \mathcal{O}(\boldsymbol{\mu})\right)=0$.

From Corollary 3.3 and Conjecture 6.2 we deduce

Corollary 6.3. For any multipartition $\boldsymbol{\mu}$ we have $\left[\Gamma\left(\mathcal{B}_{N}^{r}, \operatorname{Sym}^{\bullet} \mathcal{T}_{r} \mathcal{B}_{N}^{r} \otimes\right.\right.$ $\mathcal{O}(\boldsymbol{\mu}))]=\sum_{\boldsymbol{\lambda} \geq \boldsymbol{\mu}} K_{\boldsymbol{\lambda} \boldsymbol{\mu}}\left(t_{1}, \ldots, t_{r}\right) \chi^{\boldsymbol{\lambda}}$. Hence for any multipartitions $\boldsymbol{\lambda} \geq \boldsymbol{\mu}$ we have $K_{\boldsymbol{\lambda} \boldsymbol{\mu}}\left(t_{1}, \ldots, t_{r}\right) \in \mathbb{N}\left[t_{1}, \ldots, t_{r}\right]$.

\section{Added in Proof}

Conjecture 2.4 is proved in [17]. Conjecture 6.2 follows from 15] (see [6]). We are grateful to Wen-Wei Li and Yue Hu for bringing [15] to our attention. 


\section{References}

1. P. Achar, An implementation of the generalized Lusztig-Shoji algorithm, https://www.math.lsu.edu/pramod/docs/lsalg.pdf

2. P. Achar and A. Henderson, Orbit closures in the enhanced nilpotent cone, Adv. Math., 219 (2008), 27-62.

3. M. Brion and S. Kumar, Frobenius splitting methods in geometry and representation theory, Progress in Math. 231 Birkhäuser Boston (2005).

4. R. Brylinski, Limits of weight spaces, Lusztig's $q$-analogs, and fiberings of adjoint orbits, J. Amer. Math. Soc., 2 (1989), 517-533.

5. M. Finkelberg, V. Ginzburg and R. Travkin, Mirabolic affine Grassmannian and character sheaves, Selecta Math. (N.S.), 14 (2009), 607-628.

6. Yue Hu, Higher cohomology vanishing of line bundles on generalized Springer's resolution, arXiv:1704.07947.

7. S.-I. Kato, Spherical functions and a $q$-analogue of Kostant's weight multiplicity formula, Inv. Math., 66 (1982), 461-468.

8. G. Lusztig, Green polynomials and singularities of unipotent classes, Adv. Math., 42 (1981), 169-178.

9. G. Lusztig, Singularities, character formulas, and a $q$-analogue of weight multiplicities, Astérisque, 101-102 (1983), 208-227.

10. G. Lusztig, Canonical bases arising from quantized enveloping algebras, J. Amer. Math. Soc., 3 (1990), 447-498.

11. G. Lusztig, Quivers, perverse sheaves, and quantized enveloping algebras, J. Amer. Math. Soc., 4 (1991), 365-421.

12. I. G. Macdonald, Symmetric Functions and Hall Polynomials, 2nd ed., Oxford Mathematical Monographs, Oxford University Press, 1995.

13. O. Mathieu, Formules de caractères pour les algèbres de Kac-Moody générales, Astérisque 159-160 (1988), 1-267.

14. V. B. Mehta and W. van der Kallen, A simultaneous Frobenius splitting for closures of conjugacy classes of nilpotent matrices, Compositio Math., 84 (1992), 211-221.

15. D. Panyushev, Generalised Kostka-Foulkes polynomials and cohomology of line bundles on homogeneous vector bundles, Selecta Math. (N.S.), 16 (2010), no. 2, 315-342.

16. T. Shoji, Green functions attached to limit symbols, Adv. Stud. Pure Math., 40 (2004), 443-467.

17. T. Shoji, Kostka functions associated to complex reflection groups and a conjecture of Finkelberg-Ionov, arXiv:1702.02711.

18. R. Travkin, Mirabolic Robinson-Shensted-Knuth correspondence, Selecta Math. (N.S.) 14 (2009), 727-758. 